Case Report

\title{
Embedded Pork Bone Causing Esophageal Perforation and an Esophagus-Innominate Artery Fistula
}

\author{
Andrew C. Berry, ${ }^{1}$ Peter V. Draganov, ${ }^{2}$ Brijesh B. Patel, ${ }^{3}$ Danny Avalos, ${ }^{4}$ \\ Warren L. Reuther III, ${ }^{5}$ Avinash Ravilla, ${ }^{1}$ Bruce B. Berry, ${ }^{6}$ and Michael J. Monzel ${ }^{7}$ \\ ${ }^{1}$ Kansas City University of Medicine and Biosciences (KCUMB), 1750 E. Independence Avenue, Kansas City, MO 64106, USA \\ ${ }^{2}$ Department of Medicine and Advanced Therapeutic Endoscopy, College of Medicine, University of Florida, Gainesville, \\ Florida, FL 32610, USA \\ ${ }^{3}$ Department of Internal Medicine, University of South Florida, Tampa, FL 33612, USA \\ ${ }^{4}$ Department of Internal Medicine, University of Miami Miller School of Medicine, Palm Beach Regional Campus, \\ Atlantis, FL 33462, USA \\ ${ }^{5}$ Department of Radiology, West Palm Hospital, West Palm Beach, FL 33407, USA \\ ${ }^{6}$ Department of Internal Medicine, Wheaton Franciscan Healthcare, Milwaukee, WI 53215, USA \\ ${ }^{7}$ Digestive Disease Center of the Palm Beaches, Loxahatchee, FL 33470, USA
}

Correspondence should be addressed to Andrew C. Berry; aberry5555@gmail.com

Received 11 June 2014; Revised 19 July 2014; Accepted 21 July 2014; Published 3 August 2014

Academic Editor: Yoshihiro Moriwaki

Copyright (c) 2014 Andrew C. Berry et al. This is an open access article distributed under the Creative Commons Attribution License, which permits unrestricted use, distribution, and reproduction in any medium, provided the original work is properly cited.

Chronically embedded foreign bodies can lead to perforations, mediastinitis, and abscess, amongst a host of other complications. A 20 -year-old mentally challenged female presented with "something stuck in her throat," severe dysphagia, and recurrent vomiting. Initial imaging was unremarkable; however, subsequent imaging and esophagogastroduodenoscopy two weeks later revealed an embedded pork bone. Surgery was performed to remove the bone and fix the subsequent esophageal perforation and esophagusinnominate artery fistula. This case helps reinforce the urgency in removing an ingested foreign body and the ramifications that may arise with chronically embedded foreign bodies.

\section{Introduction}

Foreign body ingestion is very common among the pediatric population and among adults who are mentally challenged or with psychiatric illnesses [1-3]. The esophagus is the most common anatomic location for a foreign body to impact [4]. Although most foreign bodies pass spontaneously, $10 \%$ to $20 \%$ of the cases require nonoperative intervention such as rigid endoscopy or flexible endoscopy. Less than $1 \%$ will require surgical intervention [5]. Serious life-threatening complications of foreign body ingestion do occur, but in less than $1 \%$ of the cases [6]. Of these complications, esophageal perforation carries a mortality rate that remains as high as $20 \%$ and can result in serious complications such as mediastinitis and retropharyngeal or parapharyngeal abscesses [68]. We describe the management of a chronically impacted esophageal foreign body not only causing an anterior and posterior perforation but also forming an esophagus to innominate artery fistula.

\section{Case Report}

A 20-year-old mentally challenged female complained of "something stuck in her throat," severe dysphagia, and recurrent vomiting. She was seen two weeks priorly for possible pork bone ingestion; however, initial chest X-ray and soft tissue imaging of the neck were negative. Vital signs upon readmission were blood pressure of $106 / 80$, heart rate of 131 beats per minute, respiratory rate of 20 , temperature of $37.0^{\circ} \mathrm{C}$, and an oxygen saturation of $98 \%$ on room air. Her physical exam was unremarkable. There was no evidence of 


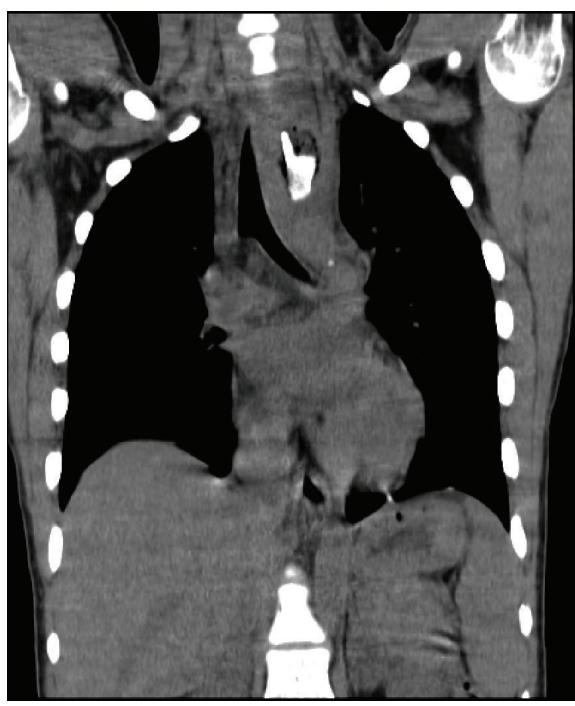

(a)

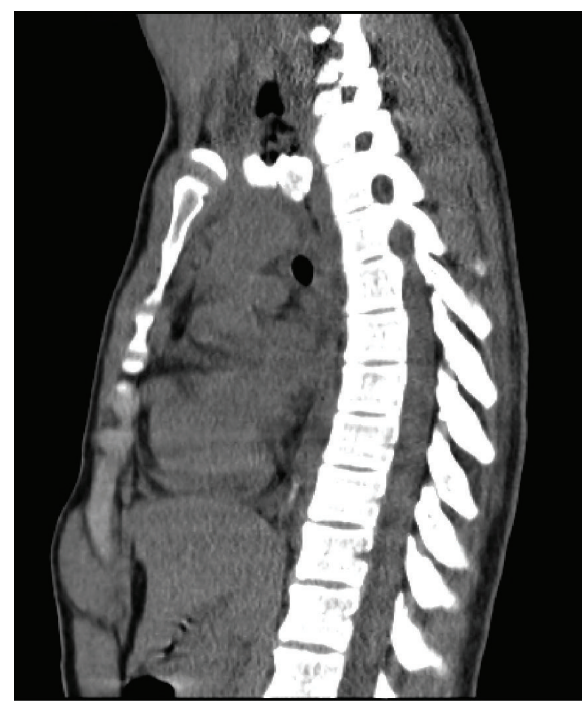

(b)

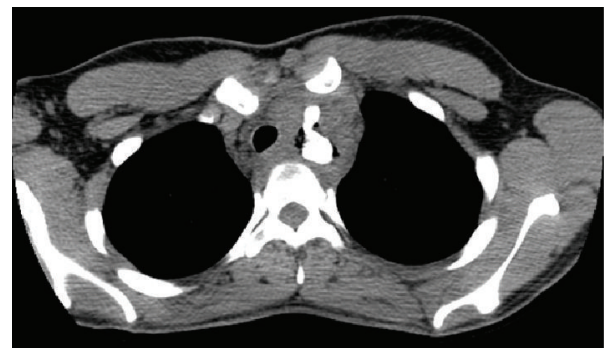

(c)

Figure 1: Computed tomography (CT) of the chest without contrast demonstrating a $3.2 \mathrm{~cm}$ (cranial/caudal) by $2.5 \mathrm{~cm}$ (anterior/posterior) Y-shaped foreign body within the anterior-superior mediastinum in the region of the esophagus. There is gas surrounding the mass. The foreign body has bone density and the gas is in part extraluminal. Edema is present in the circumference of the foreign body and is thought to shift the trachea from the left to the right, with compression of the airway. The soft tissue swelling is also evident surrounding the foreign body and esophagus, reflective of local inflammatory changes and hematoma secondary to the fistula connection between the esophagus and proximal innominate artery. (a) Coronal view. (b) Sagittal view. (c) Axial view.

subcutaneous crepitations. The patient's metabolic panel and complete blood count were within the normal range with the exception of hemoglobin $11.7 \mathrm{gm} / \mathrm{dL}$. Computed tomography (CT) of the chest without IV or oral contrast showed a large foreign body (with air density most likely representing a bone) in the proximal esophagus at the level of the thoracic inlet, with compression of the airway and tracheal deviation to the right (Figure 1). No pneumomediastinum or subcutaneous emphysema was identified.

Esophagogastroduodenoscopy (EGD) using a thin $5.5 \mathrm{~mm}$ diameter scope (Olympus, GIF XP180N) revealed a bone embedded into the esophagus (Figure 2). The scope could pass the embedded foreign body, but no attempt was made to remove it because it was difficult to ascertain the depth of penetration into the esophageal wall and the likelihood of an esophageal perforation. The patient was given antibiotics, thoracic surgery was consulted, and the patient was transferred to another hospital. Overnight the patient experienced coffee-ground emesis with occasional hematemesis.

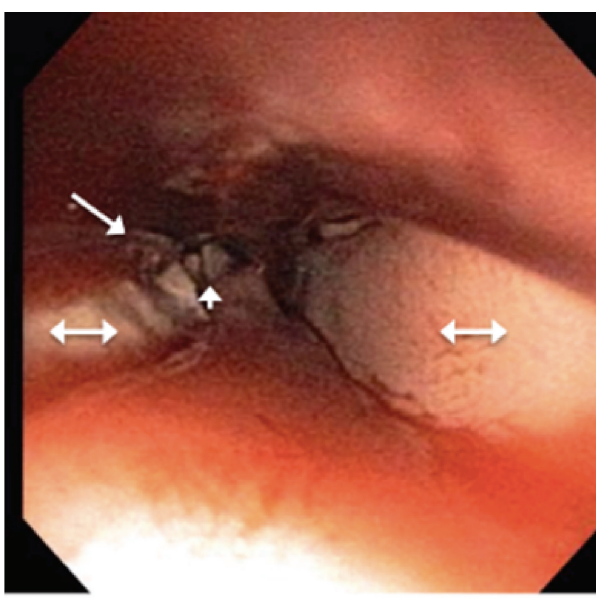

Figure 2: Esophagogastroduodenoscopy (EGD) showing a foreign body (arrowhead) embedded in the distal esophagus with ulcerations (single-sided arrow) in the surrounding mucosa, with subsequent surrounding edema (double-sided arrow). 
At the next day, a rigid esophagoscopy under general anesthesia done in the operating room showed the upper esophagus filled with dark blood; as the scope was advanced new bright red blood was encountered. A surgical incision was made along the sternocleidomastoid muscle on the left side. Within the tracheoesophageal groove, a distended and blood-filled proximal esophagus was encountered. An esophageal incision was made and brisk blood came through the esophagus and the proximal innominate artery was identified. When pressure was relieved, bleeding was noted from the back wall of the innominate artery at its junction to the aortic arch.

Perforation of the esophagus extended into the back of the innominate artery. The artery was repaired with suture and the esophageal incision was extended to the perforation. A suction catheter helped grasp and excise the dislodged $3.2 \mathrm{~cm} \times 2.5 \mathrm{~cm}$ Y-shaped bony fragment. The esophageal perforation and incision were closed with suture, a JacksonPratt drain was placed through the neck, sternotomy was closed, and a chest tube was inserted. The patient was critical throughout the procedure but was stable upon completion amid a 4-liter (L) blood loss. The patient made a gradual recovery and subsequent esophagram a week later showed no evidence of extravasation of contrast, obstruction, or strictures.

\section{Discussion}

Although the majority of foreign body ingestions can be managed conservatively and pass on their own through the GI tract, some cases require further endoscopic or even surgical management [5]. Foreign body esophageal impactions should be removed within 24 hours of the impaction to minimize the risk of perforation, mediastinitis, and abscess [9]. Our patient's initial CXR and soft tissue imaging of the neck were negative for any foreign body but identified two weeks later on CT of the neck and chest. Our case was refractory to all nonsurgical endoscopic intervention. It ultimately required a sternotomy to facilitate removal. The pork bone became embedded within the esophageal wall over a two-week period leading to mucosal edema and narrowing of the passageway. The chronically embedded foreign body led to an anterior and posterior esophageal perforation and an esophagusinnominate artery fistula.

Proper management of an esophageal foreign body removal depends on the size, location, and degree of tissue penetration. If plain films do not successfully locate a foreign body, an esophagram with barium or gastrografin can be performed. However, an esophagram is not recommended due to its increased risk of aspiration [10] and by its high reported false-positive and false-negative rates [11]. CT has been shown to be most sensitive for localizing esophageal foreign bodies and provides superior benefit to other modalities in locating additional complications, such as esophageal perforations [12]. If the foreign body does not pass quickly on its own, endoscopic management is warranted. This typically involves endoscopic rat-tooth forceps. In selected cases a dual-channel endoscope may be more advantageous than a standard flexible endoscope because of its greater diameter and ability to be used with a balloon catheter to minimize mucosal damage by expanding the esophagus [13]. If severe risk of bleeding or perforation is evident, as in our case, no further endoscopic evaluation should occur and urgent surgical evaluation is warranted to remove the foreign body and manage any bleeding or perforations. Had the foreign body been removed endoscopically and esophageal perforation was later noted, endoscopically placed removable esophageal stents, combined with aggressive conservative therapy, remain the standard of care [14].

Our case is unique because the foreign body remained embedded in the esophagus for an extended period of time making its removal an arduous task amid an esophagusinnominate artery fistula and an esophageal perforation. This case helps reinforce the urgency in removing an ingested foreign body and the ramifications that may arise with chronically embedded foreign bodies. Proper imaging workup and management must be swiftly initiated to help identify any foreign bodies and any associated tissue changes.

\section{Disclosure}

No financial support was obtained for the project. No financial grant was obtained. None of the authors listed received any financial support or services or any other contributions for their work. Informed patient consent was obtained and all patient identifiers have been removed.

\section{Conflict of Interests}

The authors declare that there is no conflict of interests regarding the publication of this paper.

\section{Authors' Contribution}

All authors contributed to evaluating and/or managing the case and to writing/editing the paper. AC Berry is the paper guarantor.

\section{References}

[1] S. Hachimi-Idrissi, L. Corne, and Y. Vandenplas, "Management of ingested foreign bodies in childhood: our experience and review of the literature," European Journal of Emergency Medicine, vol. 5, no. 3, pp. 319-323, 1998.

[2] K. E. Blaho, K. S. Merigian, S. L. Winbery, L. J. Park, and M. Cockrell, "Foreign body ingestions in the emergency department: case reports and review of treatment," The Journal of Emergency Medicine, vol. 16, pp. 21-22, 1998.

[3] I. Kamal, J. Thompson, and D. M. Paquette, "The hazards of vinyl glove ingestion in the mentally retarded patient with pica: new implications for surgical management," Canadian Journal of Surgery, vol. 42, no. 3, pp. 201-204, 1999.

[4] Z. S. Li, Z. X. Sun, D. W. Zou, G. M. Xu, R. P. Wu, and Z. Liao, "Endoscopic management of foreign bodies in the upperGI tract: experience with 1088 cases in China," Gastrointestinal Endoscopy, vol. 64, no. 4, pp. 485-492, 2006. 
[5] G. M. Eisen, T. H. Baron, J. A. Dominitz et al., "Guideline for the management of ingested foreign bodies," Gastrointestinal Endoscopy, vol. 55, no. 7, pp. 802-806, 2002.

[6] J. S. An, I. H. Baek, S. Y. Chun, and K. O. Kim, "Successful endoscopic band ligation of esophageal perforation by fish bone ingestion," Journal of Laparoendoscopic \& Advanced Surgical Techniques A, vol. 23, no. 5, pp. 459-462, 2013.

[7] P. Bhatia, D. Fortin, R. I. Inculet, and R. A. Malthaner, "Current concepts in the management of esophageal perforations: a twenty-seven year Canadian experience," Annals of Thoracic Surgery, vol. 92, no. 1, pp. 209-215, 2011.

[8] P. Ryom, J. B. Ravn, L. Penninga et al., "Aetiology, treatment and mortality after oesophageal perforation in Denmark," Danish Medical Bulletin, vol. 58, no. 5, 2011.

[9] K. L. Anderson and A. J. Dean, "Foreign bodies in the gastrointestinal tract and anorectal emergencies," Emergency Medicine Clinics of North America, vol. 29, no. 2, pp. 369-400, 2011.

[10] O. Ekberg, "Normal anatomy and techniques of examination of the esophagus: fluoroscopy, CT, MRI, and scintigraphy," in Margulis and Burhennes's Alimentary Tract Radiology, P. C. Freeny and G. W. Stevenson, Eds., p. 183, Mosby, St. Louis, Mo, USA, 1994.

[11] A. Derowe and D. Ophir, "Negative findings of esophagoscopy for suspected foreign bodies," The American Journal of Otolaryngology_Head and Neck Medicine and Surgery, vol. 15, no. 1, pp. 41-45, 1994.

[12] E. M. de Lucas, P. Sádaba, P. L. García-Barón et al., "Value of helical computed tomography in the management of upper esophageal foreign bodies," Acta Radiologica, vol. 45, no. 4, pp. 369374, 2004.

[13] C. Wang and P. Chen, "Removal of impacted esophageal foreign bodies with a dual-channel endoscope: 19 cases," Experimental and Therapeutic Medicine, vol. 6, no. 1, pp. 233-235, 2013.

[14] K. Ben-David, K. Behrns, S. Hochwald, G. Rossidis, A. Caban, and C. Crippen, "Discussion," Journal of the American College of Surgeons, vol. 218, no. 4, pp. 774-775, 2014. 


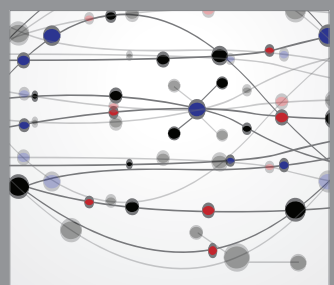

The Scientific World Journal
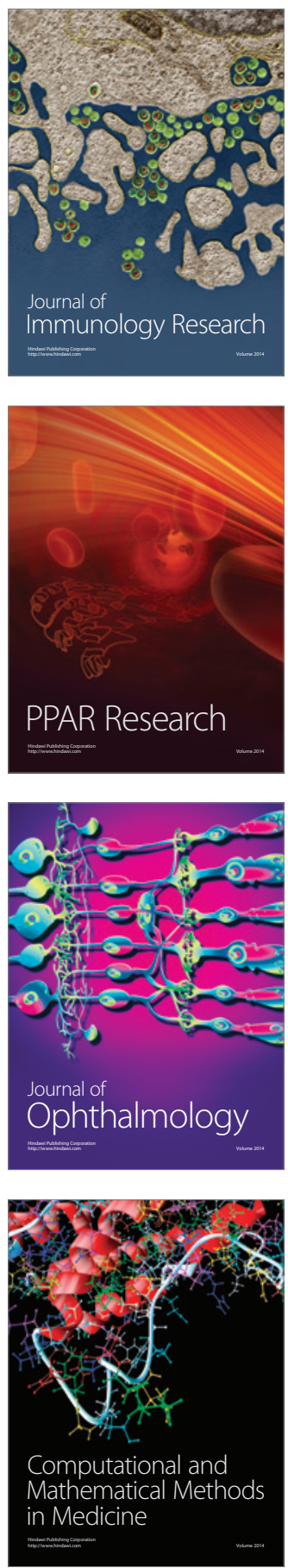

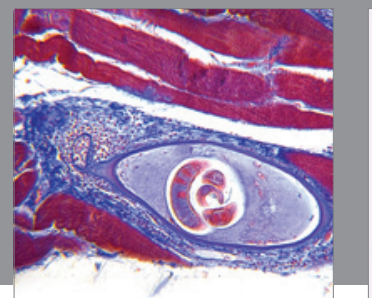

Gastroenterology

Research and Practice
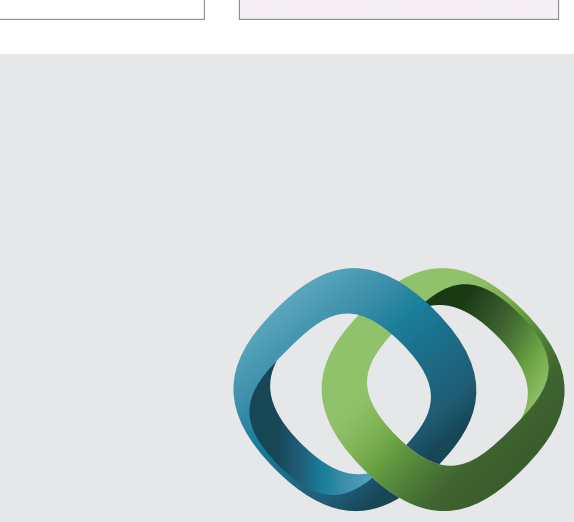

\section{Hindawi}

Submit your manuscripts at

http://www.hindawi.com
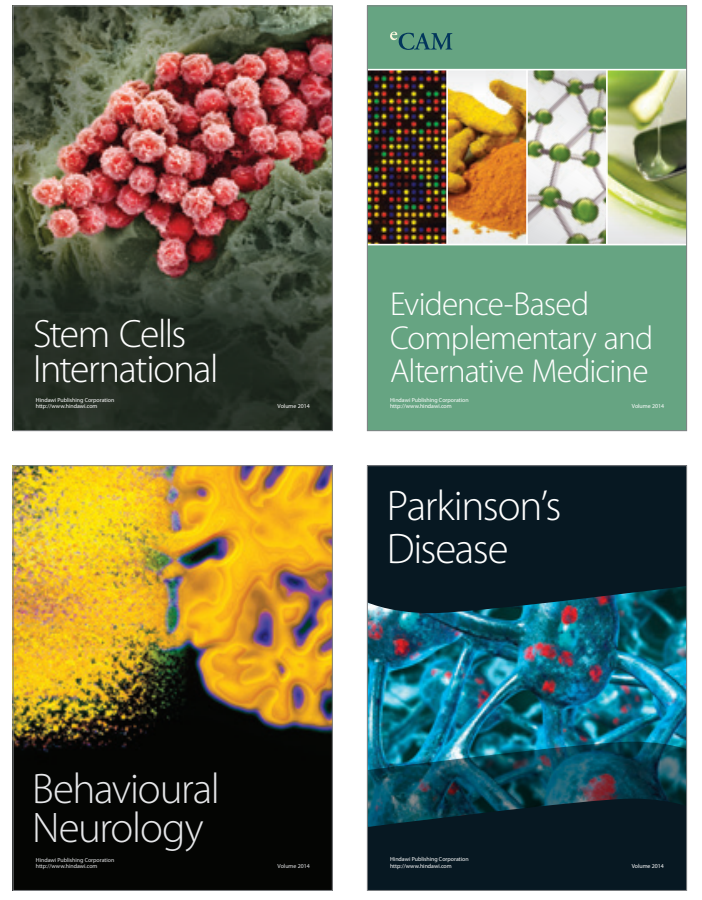
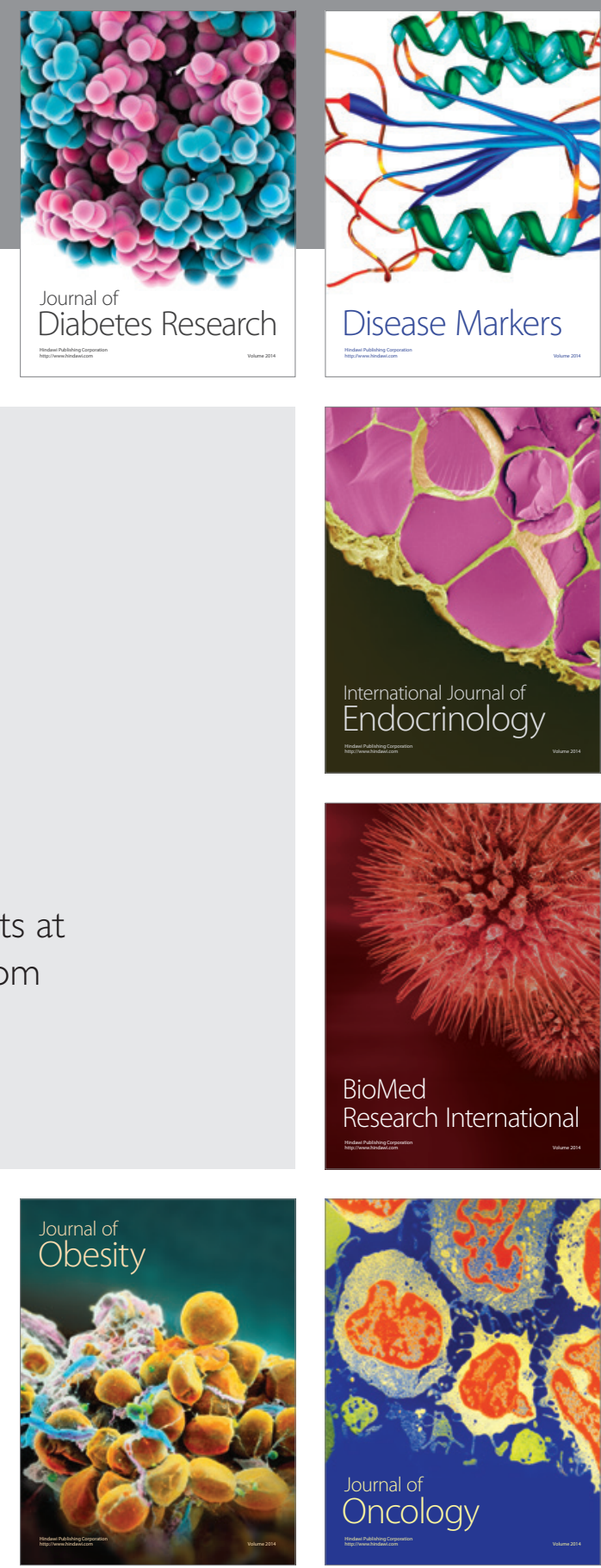

Disease Markers
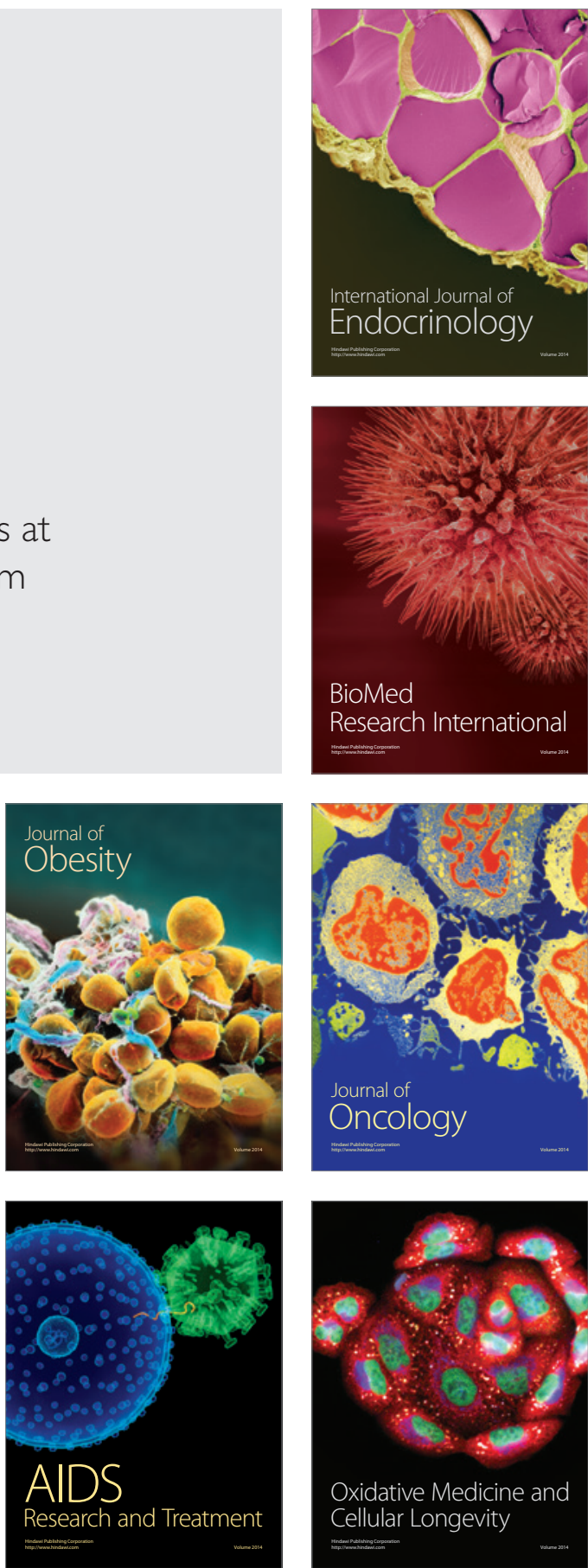